\title{
NONEXISTENCE OF H-CONVEX CUSPIDAL STANDARD FUNDAMENTAL DOMAIN
}

\author{
OMER YAYENIE
}

\begin{abstract}
It is well-known that if a convex hyperbolic polygon is constructed as a fundamental domain for a subgroup of the modular group, then its translates by the group elements form a locally finite tessellation and its side-pairing transformations form a system of generators for the group. Such hyperbolically convex polygons can be obtained by using Dirichlet's and Ford's polygon constructions. Another method of obtaining a fundamental domain for subgroups of the modular group is through the use of a right coset decomposition and we call such domains standard fundamental domains. In this paper we give subgroups of the modular group which do not have hyperbolically convex standard fundamental domain containing only inequivalent cusps.
\end{abstract}

\section{Introduction}

Let $\Gamma(1)$ denote the inhomogeneous modular group acting on the upper half plane $\mathbb{H}$ in the usual manner:

$$
\tau \stackrel{M}{\longmapsto} \frac{a \tau+b}{c \tau+d}, \quad a, b, c, d \in \mathbb{Z}, \quad a d-b c=1 .
$$

We shall denote the above mapping by its matrix form

$$
M=\left(\begin{array}{cc}
a & b \\
c & d
\end{array}\right) \text {. }
$$

Note that $M$ and $-M$ define the same mapping in $\Gamma(1)$. The modular group $\Gamma(1)$ is generated by the transformations (see $[6], \S 1.2$ )

$$
S=\left(\begin{array}{ll}
1 & 1 \\
0 & 1
\end{array}\right): \tau \stackrel{S}{\longmapsto} \tau+1 \quad \text { and } \quad T=\left(\begin{array}{cc}
0 & -1 \\
1 & 0
\end{array}\right): \tau \stackrel{T}{\longmapsto} \frac{-1}{\tau}
$$

satisfying the relations (as mappings)

$$
T^{2}=(S T)^{3}=I .
$$

Received January 15, 2008; Revised June 30, 2009.

2000 Mathematics Subject Classification. Primary 11F06, 11F03, 20H05, $20 \mathrm{H} 10$.

Key words and phrases. modular group, congruence subgroup, fundamental domain, hyperbolic convexity. 
Among the subgroups of $\Gamma(1)$ the congruence subgroups such as

$$
\begin{aligned}
\Gamma(N) & :=\{M \in \Gamma(1): a \equiv d \equiv 1(\bmod N), b \equiv c \equiv 0(\bmod N)\}, \\
\Gamma_{0}(N) & :=\{M \in \Gamma(1): c \equiv 0(\bmod N)\}, \\
\Gamma^{0}(N) & :=\{M \in \Gamma(1): b \equiv 0(\bmod N)\}=T \Gamma_{0}(N) T^{-1}
\end{aligned}
$$

have been the objects of detailed studies due to their significance in modular forms and other topics in analytic number theory. In this paper we are concerned with subgroups $\Gamma$ of $\Gamma(1)$ with finite index $([\Gamma(1): \Gamma]<\infty)$.

As a group of transformations on the upper half-plane $\mathbb{H}, \Gamma(1)$ is a discontinuous group. It has a fundamental domain (we are using Lehner's definition, see [5], $§ \mathrm{IV} .1), \operatorname{int}(\mathbb{F})$, where $\mathbb{F}$ is given by

$$
\mathbb{F}=\left\{\tau \in \mathbb{H}:|\Re(\tau)| \leq \frac{1}{2},|\tau| \geq 1\right\} .
$$

Also, if $M \in \Gamma(1)$, then the interior of $M(\mathbb{F})$ is a fundamental domain of $\Gamma(1)$. Figure 1 shows how the regions $M(\mathbb{F})$ (in the figure $M$ stands for the triangle $M(\mathbb{F}))$ fit together to form a tessellation of the upper half-plane. Moreover, two translates of $\mathbb{F}$, say $M(\mathbb{F})$ and $N(\mathbb{F})$, have a common side if and only if $M=N T, M=N S$, or $M=N S^{-1}$. If $M(\mathbb{F})$ and $N(\mathbb{F})$ are adjacent(or have a common side) and $M(\infty) \neq N(\infty)$, then $M=N T$.

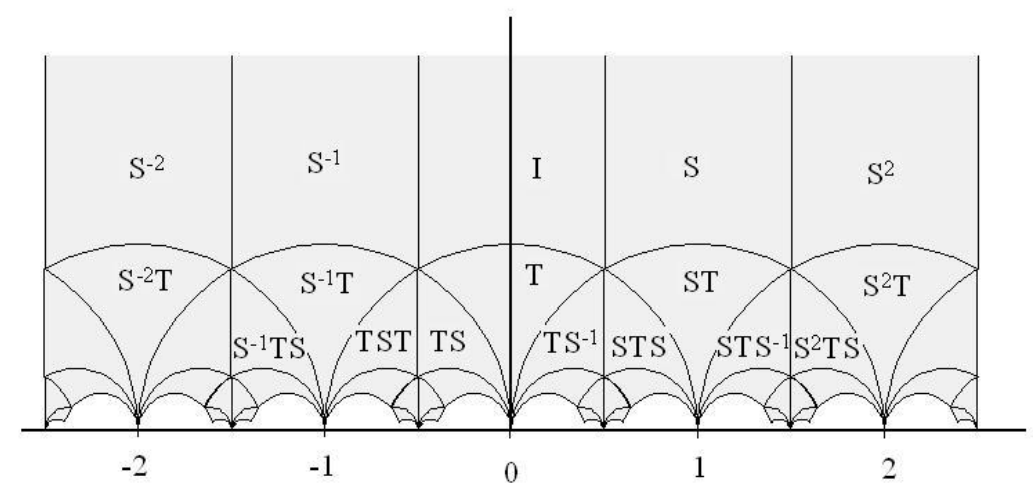

Figure 1. Tessellation of the upper half plane

Suppose that $\Gamma$ is a subgroup of $\Gamma(1)$ of index $\mu$. Then $\Gamma$ is a discontinuous group on the upper half-plane and has a fundamental domain. One way of obtaining a fundamental domain is through the use of a right coset decomposition. There is a fundamental domain for $\Gamma$ consisting of $\mu$ translates of $\mathbb{F}$. If $\Gamma(1)=\Gamma \cdot\left\{L_{1}, L_{2}, \ldots, L_{\mu}\right\}$, is a right coset decomposition, then the set

$$
\mathcal{R}_{\Gamma}=\left(\bigcup_{k=1}^{\mu} L_{k}(\mathbb{F})\right)^{o}
$$


is a fundamental domain of $\Gamma$. Fundamental domains obtained by using right coset decomposition are called standard fundamental domains. Zagier [9] used this approach to give an explicit formula for the Petersson norm of a cusp form. There are other methods of constructing fundamental domains, namely, Ford's isometric circle method and Dirichlet's polygon construction (see [5], Chapter IV). These two methods yield hyperbolically convex (h-convex) fundamental domains. But, the standard fundamental domain $\mathcal{R}_{\Gamma}$ might not have desirable topological properties like connectedness and h-convexity. However, it is shown in [6] that the right coset representatives can be chosen suitably to make the standard fundamental domain $\mathcal{R}_{\Gamma}$ connected. It is also shown in [8] that if $\Gamma$ is a normal subgroup of $\Gamma(1)$, then the right coset representatives can be chosen suitably to make $\mathcal{R}_{\Gamma}$ h-convex.

Given an h-convex standard fundamental domain for a subgroup of the modular group, can it be constructed using Ford's and/or Dirichelt's method? The answer is no. Figure 2(a) below is a standard fundamental domain for $\Gamma(3)$ that is not a Dirichlet polygon and Figure 2(b) shows a standard fundamental domain for $\Gamma(3)$ that is not a Ford polygon.

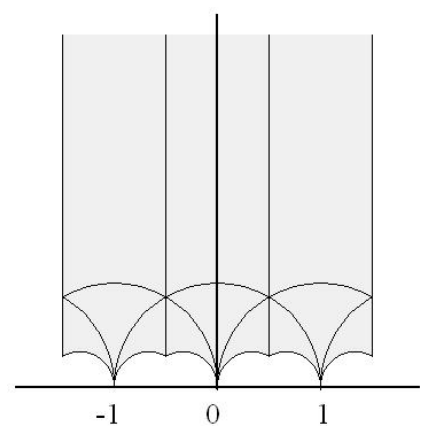

(a)

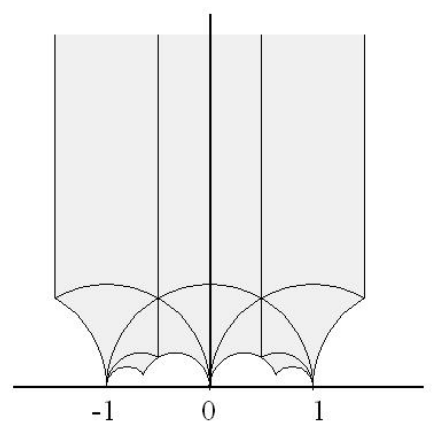

(b)

Figure 2. Standard Fundamental Domains for $\Gamma(3)$

Moreover, Ford's and Dirichlet's polygon for a subgroup $\Gamma$ of the modular group might contain two or more $\Gamma$-equivalent cusps (a cusp of $\Gamma \subseteq \Gamma(1)$ is a $\Gamma$-orbit in $\mathbb{Q} \cup\{\infty\}$, see [5] for precise definition and detailed discussions). The fundamental domain shown in Figure 3 is both a Ford and Dirichlet polygon for the subgroup $\Gamma_{0}(3)$. However, this fundamental domain contains two cusps 0 and 1 that are equivalent under $\Gamma_{0}(3)$.

Recently Kulkarni [4] constructed fundamental domains using Farey symbols which have the least number of sides. However, these fundamental domains contain almost always equivalent cusps. Figure 4 given below is a fundamental domain for $\Gamma_{0}(11)$ constructed using Kulkarni's method. This fundamental domain contains the equivalent cusps $0, \frac{1}{3}, \frac{1}{2}, \frac{2}{3}$, and 1 . 


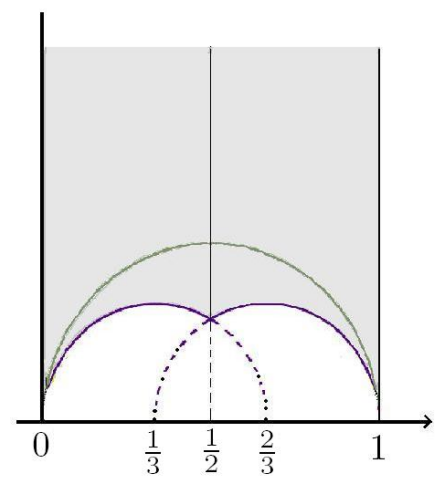

Figure 3. Dirichlet \& Ford Polygon for $\Gamma_{0}(3)$

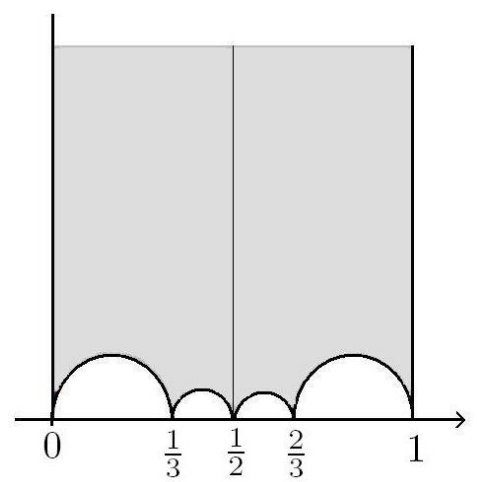

Figure 4. Fundamental Domain for $\Gamma_{0}(11)$

As we pointed out earlier, Zagier (see [9], p. 377) gave an explicit standard fundamental domain for $\Gamma^{0}(p)$, where $p$ is prime, to calculate the Petersson norm of a cusp form of a certain weight. These domains are h-convex standard fundamental domains containing only inequivalent cusps. For any prime $p$, the index of the subgroup $\Gamma^{0}(p)$ in $\Gamma(1)$ is $p+1$ and

$$
\Gamma(1)=\Gamma^{0}(p) \cdot\left\{T, I, S, \ldots, S^{p-1}\right\} .
$$

The standard fundamental domain obtained by using the above right coset decomposition (due to Zagier)

$$
\mathcal{R}_{\Gamma^{0}(p)}=\left(T(\mathbb{F}) \cup \bigcup_{j=0}^{p-1} S^{j}(\mathbb{F})\right)^{o}
$$


is h-convex and contains only two inequivalent cusps of $\Gamma^{0}(p)$, namely $0=T(\infty)$ and $\infty=I S^{j}(\infty)$ for all $j=0,1, \ldots, p-1$. Figure 5 shows an h-convex standard fundamental domain for $\Gamma^{0}(5)$ containing only inequivalent cusps.

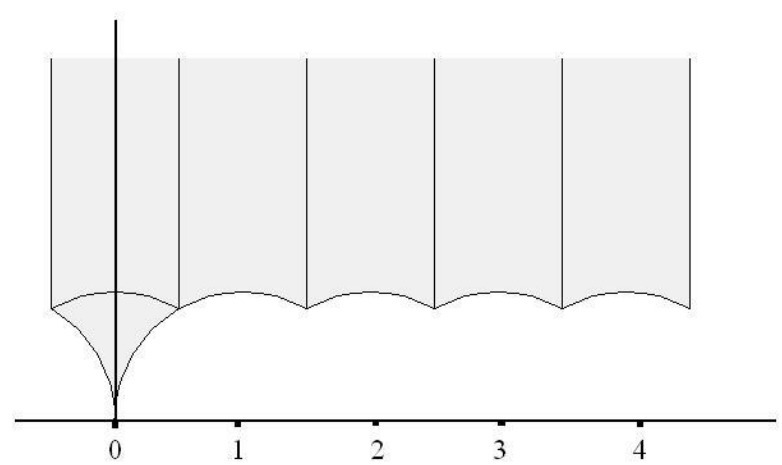

Figure 5. Fundamental Domain for $\Gamma^{0}(5)$

Following Zagier's approach one can show that the right coset decomposition

$$
\Gamma(1)=\Gamma^{0}\left(p^{2}\right)\left\{I, S, \ldots, S^{p^{2}-1}, T, S^{p} T, \ldots, S^{(p-1) p} T\right\}
$$

yields an h-convex standard fundamental domain containing only inequivalent cusps for $\Gamma^{0}\left(p^{2}\right)$ as shown in Figure 6 for $\Gamma^{0}(9)$.

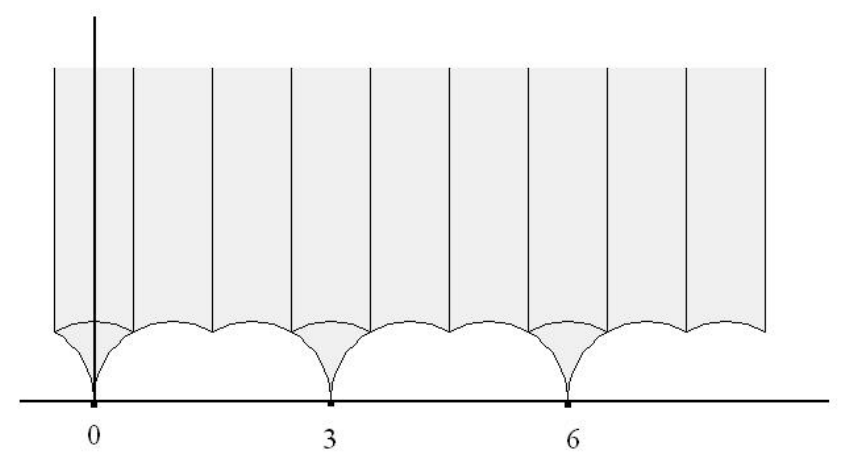

Figure 6. Fundamental Domain for $\Gamma^{0}(9)$

Other than the above constructions for special subgroups $\Gamma^{0}(N)$, where $N$ is either a prime or a square of a prime, no general method for constructing an h-convex standard fundamental domain containing only inequivalent cusps 
for subgroups of $\Gamma(1)$ seems to be discussed or to be known. It is the object of this article to show that it is not always possible to have an h-convex standard fundamental domain containing only inequivalent cusps.

\section{The result}

If there is an h-convex standard fundamental domain containing only inequivalent cusps for a subgroup $\Gamma$ of the modular group, then the right coset must have the following form. If $\Gamma$ a subgroup of $\Gamma(1)$ of index $\mu$, then there exists a finite number of elements $A_{1}, \ldots, A_{p}$ such that

$$
\Gamma(1)=\bigcup_{k=1}^{p} \bigcup_{j=0}^{\lambda_{k}-1} \Gamma A_{k} S^{j}
$$

and the set

$$
\mathcal{R}_{\Gamma}=\left(\bigcup_{k=1}^{p} \bigcup_{j=0}^{\lambda_{k}-1} A_{k} S^{j}(\mathbb{F})\right)^{o}
$$

is a standard fundamental domain for $\Gamma$, where

$$
\lambda_{k}=\min \left\{m \in \mathbb{N}: S^{m} \in A_{k}^{-1} \Gamma A_{k}\right\} .
$$

We call the interior of the set $\mathcal{R}_{\Gamma}$ a Cuspidal Standard Fundamental Domain(CSFD) for $\Gamma$, since $\mathcal{R}_{\Gamma}$ contains only inequivalent cusps. The natural number $\lambda_{k}$ is called the width of the cusp $A_{k}(\infty)$ and it counts the number of $\Gamma$-inequivalent copies of $\mathbb{F}$ attached at the point $A_{k}(\infty)$. We also say that $\Gamma$ has cusp split given by $\mu=\sum_{k=1}^{p} \lambda_{k}$. In terms of the elements of $\Gamma$, there are $p$ disjoint conjugacy classes of parabolic elements in $\Gamma$ of the form

$$
\left\{M\left\langle A_{k} S^{\lambda_{k}} A_{k}^{-1}\right\rangle M^{-1}: M \in \Gamma\right\} .
$$

The vertices of $\mathcal{R}_{\Gamma}$ lying on the boundary of the upper half-plane are the points given by $A_{k}(\infty)$ for $k=1,2, \ldots, p$, which are $\Gamma$-inequivalent cusps. The natural number $p$, given above, is called the parabolic class number. From the above description, it is not hard to see that if two cusps are $\Gamma$-equivalent, then they have the same cusp width.

Now we are ready to state the question raised by Leon Ehrenpreis and Marvin I. Knopp in a Number Theory Seminar at Temple University in Philadelphia. Is it possible to choose the set $\left\{A_{1}, \ldots, A_{p}\right\}$ suitably to make the cuspidal standard fundamental domain $\mathcal{R}_{\Gamma}$ h-convex? (Note that the fundamental domains constructed by Ford, Dirichlet, and Kulkarni are all h-convex.) The answer is a resounding no. We will give an infinite collection of subgroups of $\Gamma(1)$ which do not have an h-convex cuspidal standard fundamental domain.

Theorem 2.1. For any odd prime $p$, the congruence subgroup $\Gamma^{0}\left(p^{3}\right)$ of $\Gamma(1)$ defined by

$$
\Gamma^{0}\left(p^{3}\right)=\left\{\left(\begin{array}{ll}
a & b \\
c & d
\end{array}\right) \in \Gamma(1): p^{3} \mid b\right\}
$$


has no h-convex cuspidal standard fundamental domain.

We need the following lemmas to prove the theorem.

Lemma 2.2. Let $P$ be a hyperbolic polygon with interior angles

$$
\theta_{1}, \theta_{2}, \theta_{3}, \ldots, \theta_{n}
$$

Then $P$ is h-convex if and only if each $\theta_{k}$ satisfies $0 \leq \theta_{k} \leq \pi$.

The proof of the above lemma can be found in [1] (§7.16) and [7].

Lemma 2.3. Let $N$ be a natural number. Then

(i) $\left[\Gamma(1): \Gamma^{0}(N)\right]=N \prod_{p \mid N}\left(1+\frac{1}{p}\right)$.

(ii) A set of inequivalent cusps for $\Gamma^{0}(N)$ is given by

$$
P(N)=\left\{\frac{v}{u}:(u, v)=1, \quad v \mid N, u\left(\bmod \operatorname{gcd}\left(v, \frac{N}{v}\right)\right)\right\}
$$

and the cusp width of $\frac{v}{u}$ is given by $\frac{N}{\operatorname{gcd}\left(v^{2}, N\right)}$.

(iii) The number of inequivalent cusps for $\Gamma^{0}(N)$ is given by $\sum_{v \mid N} \phi(\operatorname{gcd}(v, N / v))$.

Since $\Gamma^{0}(N)=T \Gamma_{0}(N) T^{-1}$, the proof of the above lemma can be easily derived from Propositions 2.5 and 2.6 of [3] in $\S 2.4$. The proof of the following lemma is omitted.

Lemma 2.4. If two reduced rational numbers $\frac{a}{b}$ and $\frac{c}{d}$ are equivalent under the group $\Gamma^{0}(n)$, then $(a, n)=(c, n)$.

Proof of Theorem 2.1. First note that by Lemma 2.3 we have $\left[\Gamma(1): \Gamma^{0}\left(p^{3}\right)\right]=$ $p^{3}+p^{2}=p^{2}(p+1)$ and there are $2 p$ inequivalent cusps of $\Gamma^{0}\left(p^{3}\right)$. Moreover, the inequivalent cusps and their width are given below.

\begin{tabular}{|c|c|c|}
\hline $\begin{array}{c}\text { Index } \\
k\end{array}$ & $\begin{array}{c}\text { Cusp } q_{k} \text { belongs } \\
\text { to the orbit }\end{array}$ & $\begin{array}{c}\text { Cusp width } \\
\lambda_{k}\end{array}$ \\
\hline 1 & {$[\infty]$} & $p^{3}$ \\
\hline 2 & {$[p]$} & $p$ \\
3 & {$[2 p]$} & $p$ \\
$\vdots$ & $\vdots$ & $\vdots$ \\
$p$ & {$[(p-1) p]$} & $p$ \\
\hline$p+1$ & {$\left[p^{2}\right]$} & 1 \\
$p+2$ & {$\left[2 p^{2}\right]$} & 1 \\
$\vdots$ & $\vdots$ & $\vdots$ \\
$2 p$ & {$\left[p^{3}\right]$} & 1 \\
\hline
\end{tabular}


We prove the theorem by contradiction. Suppose there are $2 p$-transformations $A_{1}, \ldots, A_{2 p}$ such that

$$
\Gamma(1)=\Gamma^{0}\left(p^{3}\right) \cdot \bigcup_{k=1}^{2 p}\left\{A_{k}, A_{k} S, \ldots, A_{k} S^{\lambda_{k}-1}\right\}
$$

and the interior of

$$
\mathcal{R}=\bigcup_{k=1}^{2 p} \bigcup_{j=0}^{\lambda_{k}-1} A_{k} S^{j}(\mathbb{F})
$$

is an h-convex cuspidal standard fundamental domain for $\Gamma^{0}\left(p^{3}\right)$. Let $A_{k}=$ $\left(\begin{array}{ll}a_{k} & b_{k} \\ c_{k} & d_{k}\end{array}\right)$ for $k=1,2, \ldots, 2 p$. By renumbering, if necessary, we can assume that $q_{k}=A_{k}(\infty)=\frac{a_{k}}{c_{k}}$ for $k=1, \ldots, 2 p$. By Lemma 2.4, we have

$$
\left(a_{k}, p^{3}\right)= \begin{cases}1 & \text { if } k=1 \\ p & \text { if } 2 \leq k \leq p \\ p^{2} & \text { if } p<k<2 p \\ p^{3} & \text { if } k=2 p .\end{cases}
$$

If we let

$$
\mathcal{R}_{k}=\bigcup_{j=0}^{\lambda_{k}-1} A_{k} S^{j}(\mathbb{F})
$$

for $k=1,2, \ldots, 2 p$, then $\mathcal{R}_{k}$ is an h-convex polygon and

$$
\mathcal{R}=\bigcup_{k=1}^{2 p} \mathcal{R}_{k}
$$

Since the image of a fundamental domain for a group by an element of the group is also a fundamental domain, we can assume, without loss of generality, that $q_{1}=\infty$ and $A_{1}=S^{m}$ for some $m \in \mathbb{Z}$ with $-p^{3}<m \leq 0$. Therefore the h-convex polygon $\mathcal{R}_{1}$ containing $p^{3}$ translates of $\mathbb{F}$ has the form depicted below in Figure 7.

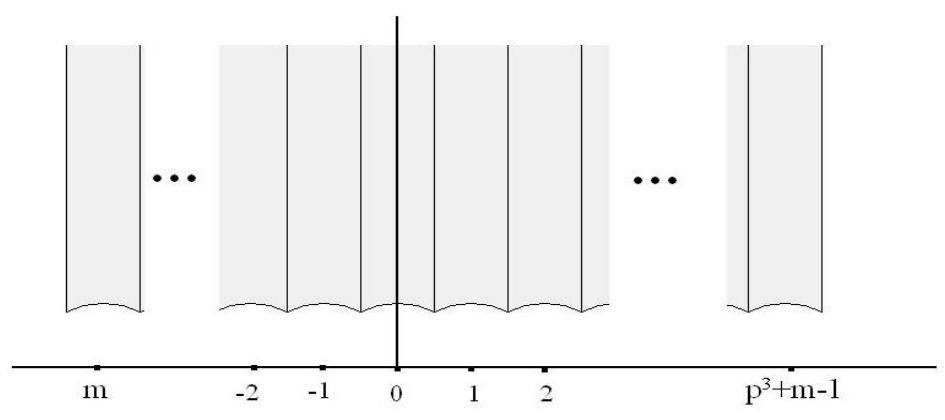

FiguRE $7 . \mathcal{R}_{1}$ 
We want to show that each of the $2 p-1$ polygons $\mathcal{R}_{2}, \ldots, \mathcal{R}_{2 p}$ shares a side with only the polygon $\mathcal{R}_{1}$. Since $\mathcal{R}$ is h-convex, connected, and a union of $2 p$ polygons, for each $n(2 \leq n \leq 2 p) \mathcal{R}_{n}$ must share a side with $\mathcal{R}_{k}$ for some $k \neq n$. We want to show that $k=1$.

Case 1: $2 \leq n \leq p$.

Since $\mathcal{R}_{n}$ shares a side with $\mathcal{R}_{k}$, there exist $\alpha_{k}$ with $0 \leq \alpha_{k}<\lambda_{k}$ and $\alpha_{n}$ with $0 \leq \alpha_{n}<p$ such that $A_{n} S^{\alpha_{n}}(\mathbb{F})$ shares a side with $A_{k} S^{\alpha_{k}}(\mathbb{F})$. Since $A_{n} S^{\alpha_{n}}(\infty)=q_{n} \neq q_{k}=A_{k} S^{\alpha_{k}}(\infty)$, we have $A_{n} S^{\alpha_{n}}=A_{k} S^{\alpha_{k}} T$ and as a result

$$
A_{n}=A_{k} S^{\alpha_{k}} T S^{-\alpha_{n}}=\left(\begin{array}{cc}
b_{k}+\alpha_{k} a_{k} & * \\
d_{k}+\alpha_{k} c_{k} & *
\end{array}\right) .
$$

Note that $p \mid\left(b_{k}+\alpha_{k} a_{k}\right)$, since $p \mid a_{n}$. If $k \neq 1$, then $p \mid a_{k}$ and consequently $p \mid b_{k}$. This contradicts the fact that $\left(a_{k}, b_{k}\right)=1$. Therefore $k=1$. Hence for each $n, 2 \leq n \leq p$, the polygon $\mathcal{R}_{n}$ shares a side only with $\mathcal{R}_{1}$.

Case 2: $p+1 \leq n \leq 2 p$.

Next we want to show that the polygons $\mathcal{R}_{n}$, for $n=p+1, \ldots, 2 p$, shares a side only with $\mathcal{R}_{1}$. As before, the polygon $\mathcal{R}_{n}$ must share a side with $\mathcal{R}_{k}$ for some $k \neq n$. That means there exists $\alpha_{k}$ with $0 \leq \alpha_{k}<\lambda_{k}$ such that $A_{n}(\mathbb{F})$ shares aside with $A_{k} S^{\alpha_{k}}(\mathbb{F})$. Since $A_{n}(\infty)=q_{n} \neq q_{k}=A_{k} S^{\alpha_{k}}(\infty)$, we have $A_{n}=A_{k} S^{\alpha_{k}} T$ and as a result

$$
A_{n}=A_{k} S^{\alpha_{k}} T=\left(\begin{array}{cc}
b_{k}+\alpha_{k} a_{k} & * \\
d_{k}+\alpha_{k} c_{k} & *
\end{array}\right) .
$$

Note that $p^{2} \mid b_{k}+\alpha_{k} a_{k}$, because $p^{2} \mid\left(a_{n}, p^{3}\right)$. If $k \neq 1$, then $p \mid a_{k}$ and consequently $p \mid b_{k}$. This is a contradiction. Therefore $k=1$. Hence each of the polygons $\mathcal{R}_{k}, k=2, \ldots, 2 p$, shares a side with $\mathcal{R}_{1}$ only.

Next we would like to show that these polygons could not be put together to form an h-convex polygon which will lead us to a contradiction. Since $\mathcal{R}_{k}$ shares a side only with $\mathcal{R}_{1}$ for each $k=2,3, \ldots, 2 p$, there exist an integer $m_{k}$ with $0 \leq m_{k}<p^{3}$ and $\alpha_{k}$ with $0 \leq \alpha_{k}<\lambda_{k}$ such that $A_{k} S^{\alpha_{k}}(\mathbb{F})$ shares a side with $A_{1} S^{m_{k}}(\mathbb{F})$. Consequently, we have

$$
A_{k} S^{\alpha_{k}}=A_{1} S^{m_{k}} T
$$

and

$$
A_{k}=A_{1} S^{m_{k}} T S^{-\alpha_{k}}=S^{m+m_{k}} T S^{-\alpha_{k}}=\left(\begin{array}{cc}
m+m_{k} & * \\
1 & *
\end{array}\right) .
$$

Also note that $m_{k} \neq m_{n}$ whenever $k \neq n$. Since $p \mid a_{k}$ for each $k \in\{2,3, \ldots, 2 p\}$, $p \mid\left(m+m_{k}\right)$. If $k \neq n$ and $k, n \in\{2,3, \ldots, 2 p\}$, then $p \mid\left(m_{k}-m_{n}\right)$. That means any two distinct cusps $A_{k}(\infty)=m+m_{k}$ and $A_{n}(\infty)=m+m_{n}$, where $k$ and $n$ are greater than one, are at least $p$ units apart on the real axis. Moreover, there is at least one cusp with width greater than one that can not share a side with $\mathcal{R}_{1}$ on any side of $S^{m}(\mathbb{F})$ and $S^{p^{3}+m-1}(\mathbb{F})$. Thus, there exists at least one $t \in\{2, \ldots, p\}$ such that $m<q_{t}=A_{t}(\infty)=m+m_{t}<p^{3}+m-1$. Now 
let us look $\mathcal{R}$ near the parabolic point $q_{t}$. Depending on $\alpha_{t}, \mathcal{R}$ looks like one of the regions in Figure 8.

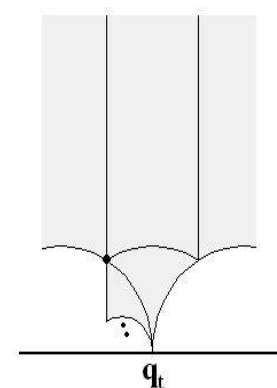

when $a_{t}=0$

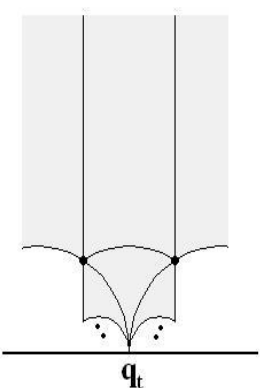

when $0<\mathrm{a}_{\mathrm{t}}<\mathrm{p}-1$

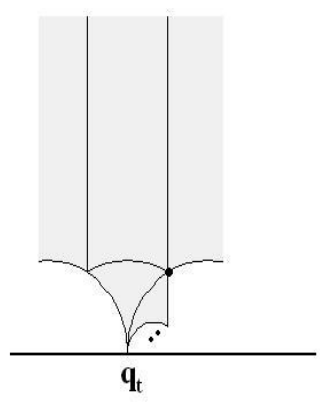

when $a_{t}=p-1$

Figure 8. $\mathcal{R}$ when zoomed in near $q_{t}$

In all of the above $p$ cases, as shown above, the interior angle at the vertex marked by a solid dot equals $4\left(\frac{\pi}{3}\right)>\pi$. This is a contradiction to the $\mathrm{h}-$ convexity of the polygon $\mathcal{R}$ (see Lemma 2.2). Therefore $\Gamma^{0}\left(p^{3}\right)$ has no h-convex cuspidal standard fundamental domain for any odd prime $p$.

Remark 2.5. In the proof of the above theorem we used the hypothesis $p \geq 3$ in order to get at least 2 inequivalent cusps of width greater than 1 . The shaded region shown in Figure 9 is an h-convex cuspidal standard fundamental domain for $\Gamma^{0}(8)$.

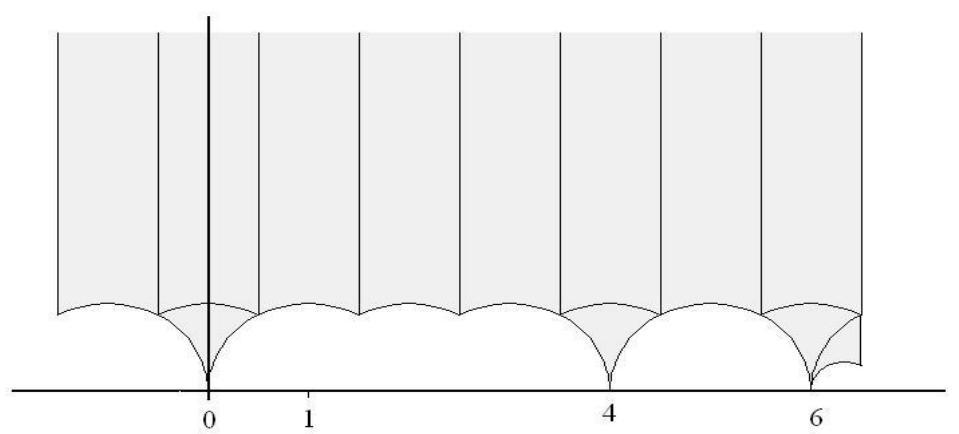

Figure 9. h-convex CSFD for $\Gamma^{0}(8)$ 


\section{References}

[1] A. F. Beardon, The Geometry of Discrete Groups, Graduate Texts in Mathematics, 91. Springer-Verlag, New York, 1983.

[2] L. Ford, Automorphic Functions, McGraw-Hill, New York, 1929.

[3] H. Iwaniec, Topics in Classical Automorphic Forms, Graduate Studies in Mathematics, 17. American Mathematical Society, Providence, RI, 1997.

[4] R. S. Kulkarni, An arithmetic-geometric method in the study of the subgroups of the modular group, Amer. J. Math. 113 (1991), no. 6, 1053-1133.

[5] J. Lehner, Discontinuous Groups and Automorphic Functions, American Mathematical Society, Providence, R.I., 1964.

[6] R. A. Rankin, The Modular Group and its Subgroups, Ramanujan Institute, Madras, 1969.

[7] H. Tietze, Über Konvexheit im kleinen und im großen und über gewisse den Punkten einer Menge zugeordnete Dimensionszahlen, Math. Z. 28 (1928), no. 1, 697-707.

[8] O. Yayenie, H-convex standard fundamental domain of a subgroup of a modular group, Ramanujan J. 16 (2008), no. 3, 305-320.

[9] D. Zagier, Modular parametrizations of elliptic curves, Canad. Math. Bull. 28 (1985), no. $3,372-384$.

Department of Mathematics \& Statistics

Murray State University

Murray, KY 42071, U.S.A.

E-mail address: omer.yayenie@murraystate.edu 